\title{
Emprendimiento: \\ Visiones desde las teorias del comportamiento humano
}

\author{
Jorge Andrés Marulanda Montoya* \\ Geovanny Correa Calle** \\ Luis Fernando Mejía Mejía***
}

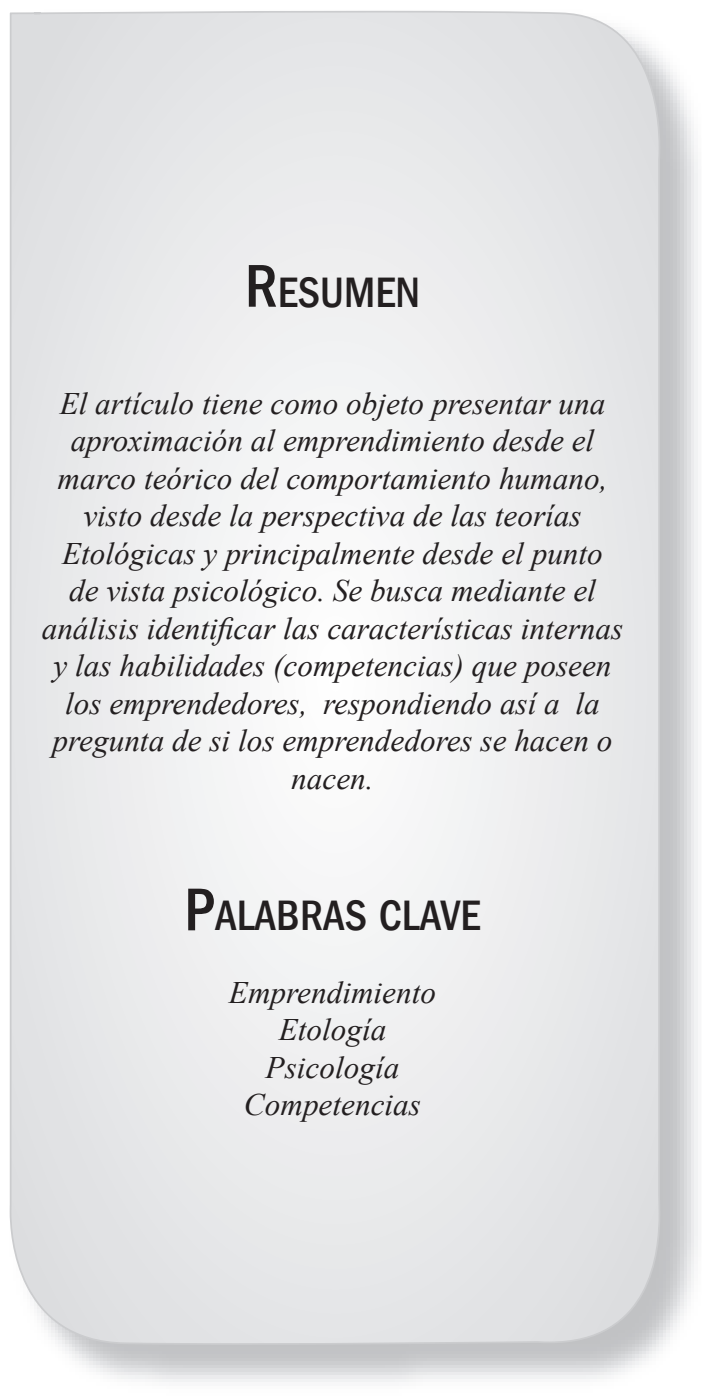

\section{Introducción}

E. emprendimiento es una de las características que determina el crecimiento, la transformación y el desarrollo de nuevos sectores económicos de una región o un país, siendo el ser humano el principal pilar. En ese sentido, se pretenden determinar las principales particularidades que permiten que unas

* Consultor Independiente, Docente Universitario Postgrado. Administrador de Empresas Ceipa. Especialista de Mercadeo Universidad Católica de Oriente. Candidato a Maestría Gestión de Organizaciones EAN - UQAC (Medellín)

** Docente Universitario Postgrado. Administrador Empresas Universidad EAFIT. Especialista Six Sigma (Cinturon negro), Candidato a Maestría Gestión de Organizaciones EAN - UQAC (Medellín)

*** Psicólogo Universidad San Buenaventura. Especialista Gerencia de Mercadeo Universidad EAFIT. Candidato a Maestría Gestión de Organizaciones EAN - UQAC (Medellín). 
personas sean más exitosas que otras en la creación e implementación de dichas ideas de emprendimiento.

El documento desarrolla una aproximación a dichas características desde el estudio del marco teórico del comportamiento humano, visto desde la perspectiva de teorías etológicas y psicológicas. Se busca mediante el análisis, identificar cómo el ser humano no es independiente a las características de su entorno, sino que también posee características internas y habilidades (competencias) que le permiten enfrentarse a nuevos retos e ideas empresariales.

A medida que se expliquen o referencien las definiciones sobre cada una de dichas teorías se presentarán los conceptos propios de los autores (inferencias) que permiten observar los elementos que hacen diferentes a un emprendedor de otros que no lo sean.

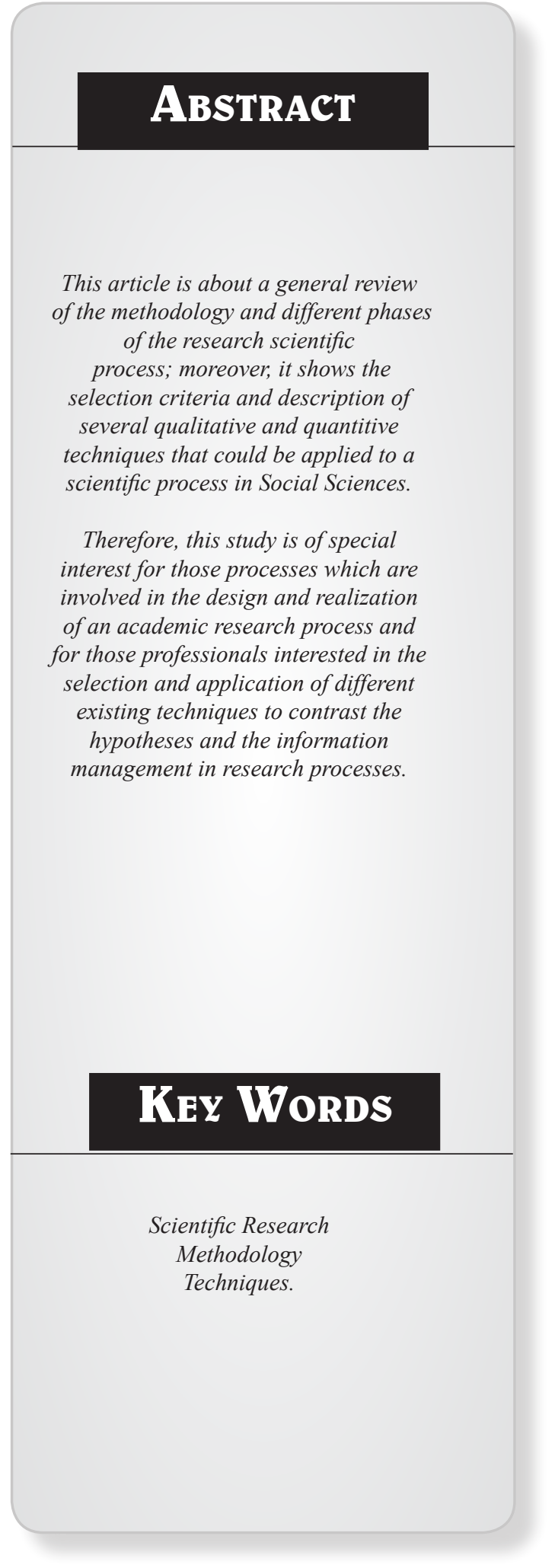




\section{El enfoque Etológico (Perinat, A., 1980)}

$\mathrm{P}$ ara lograr el objetivo del presente artículo, se debe tener claridad acerca de la terminología propia utilizada, fundamentando su orientación inicialmente en el concepto Etología, el cual se refiere al estudio biológico del comportamiento desde la perspectiva humana, por medio de la construcción de etogramas ${ }^{1}$ o repertorios detallados de pautas comportamentales, con la finalidad de brindar luces explicativas acerca de las iniciativas de emprendimiento y su relación con dicho enfoque. En ese orden de ideas, se quiere plantear una pregunta de gran relevancia a la luz de la presente teoría: - ¿Por qué el emprendedor actúa así? Dicho interrogante debe abordarse desde varias perspectivas para lograr un acercamiento adecuado, que según Timbergen (1963) se denominan los cuatro "por qué", siendo estos planteamientos, visiones desde diversos ángulos a la cuestión tratada, para llegar a su adecuado entendimiento. Los cuatro enfoques se enumeran a continuación:
D ¿Quéestímulos(internosoexternos)han desencadenado el comportamiento?

$>$ ¿Aqué propende este comportamiento, o sea, cuál es su función?

$>$ ¿Cómo este comportamiento ha surgido dentro del conjunto de pautas que caracterizan a un grupo de individuos?

D ¿Quévaloradaptativoodesupervivencia tiene este comportamiento?

El autor sustenta que la teoría evolucionista ${ }^{2}$ es la que nos da la razón de la continuidad de la vida sobre la tierra, la cual explica a la vez la diversidad de la especie $\mathrm{Homo}^{3}$, además sostiene, que el concepto de adaptación se da como instrumento de exploración del comportamiento humano y más particularmente del desarrollo. Hasta este punto, la evolución del individuo solo está tomada como el desarrollo del

\footnotetext{
La suma de las pautas de conducta propias de una especie, que le han permitido sobrevivir y llegar hasta nuestros tiempos. Diccionario canis.cl.

La teoría de Charles Darwin sobre el origen y evolución de las especies, también conocida como Darwinismo, está basada fundamentalmente en la selección natural y en la lucha por la existencia, aunque en realidad abarca otros muchos aspectos.

Es un género1 de primates homínidos de la tribu Hominini. El género Homo incluye al ser humano moderno y a sus más cercanos parientes. Fuente Wikipedia.org.
} 
ser, justificado en su comportamiento sobre el hábitat y en su intención de mejorar sus condiciones de vida, como idea de su entorno, factor que puede orientar argumentaciones acerca del bajo impacto que los emprendimientos basados en la necesidad tienen en el desarrollo económico, convirtiéndose estos básicamente en medios de supervivencia individual.

La explicación del comportamiento del ser humano, se distingue claramente de la explicación de estímulos y motivaciones como balance del intercambio organismo - medio ambiente.

Entendiendo la definición y las pautas de preguntas que propone el autor, para iniciar el diseño del análisis (inferencia) acerca de que la evolución del ser humano se ha dado producto de la constante de encontrar mejores condiciones de vida, sujetas al entorno directo donde habita y que está condicionada por las diferentes iniciativas de mejoramiento de su entorno, en la medida en que su capacidad de razonamiento se lo ha permitido. Cabe señalar que el comportamiento del ser humano hasta este punto se condiciona de acuerdo con su medio, dando por hecho que no hay nada distinto que lo mueva a mejorar su condición de vida más que el espacio donde habita.

Respondiendo las preguntas propuestas porTimbergen, desde dichas perspectivas, se entiende primero, que el ser humano se estimula por asociación de ideas, resultado de la necesidad por la supervivencia (orientado desde la capacidad del ser humano para razonar), de tal forma que una idea de emprendimiento, sin que esto haya significado una idea de empresa, busca la mejora de calidad de vida de la persona, impactando directamente sobre su hábitat. Como ejemplo de ello se podría citar al hombre prehistórico, el cual con el fin de guarecerse del invierno, aprendió a recolectar maderos secos para producir fuego dentro de las cavernas y así proveerse del calor necesario. De aquí que se pueda inferir que aquellos primeros seres humanos que lograron influenciar o liderar un grupo, podrían ser considerados una especie de emprendedores de su clan. En el anterior ejemplo se puede ver de manera análoga, como las iniciativas de emprendedores actuales, se dan hoy en la gestión efectiva de los inventarios de todas las clases con el fin de producir empleo, generar ingresos, al mismo tiempo que se optimiza el capital de trabajo.

Segundo: este comportamiento propende garantizar la función de supervivencia, sin conocimiento específico de lo que eso significaba, más que el temor innato de vivir en medio de condiciones desfavorables a su condición humana, sin que esto signifique que el ser humano en ese entonces era del todo racional como para generar ideas de mejorar su condición, lo que en principio una caverna fue la 
idea de una vivienda para guarecerse, siendo hoy uno de los mejores modelos de negocio en el mundo.

Tercero: este comportamientosurge dentro de un conjunto de pautas condicionadas por el entorno, es decir, la casuística de las condiciones del medio - invierno, verano , frio, calor, la sensación de hambre, entre otras -, inducían el comportamiento del ser humano para encontrar la forma de apropiarse de esas condiciones y favorecerse: como la caza de animales para proveerse de alimentos (sector alimenticio, uno de los mejores sectores actuales desde el cual se propenden por ideas de negocio para el mejoramiento de desarrollo económico); y cuarto, el valor de adaptarse significaba la continuidad de su especie trascendiendo sobre la capacidad de enseñar a otros desde lo aprendido, sin que esto haya significado escuela formal (otro sector desde donde se propende por generar ideas de negocios sustentadas en universidades e instituciones de aprendizaje de toda índole).

Si nos abstraemos al tema de emprendimiento, debemos reconocer que muchas de las interacciones con el entorno, han sido desarrollos no concebidos como ideas de negocio, pero que dieron pié a que ideas traídas a negocios nacen de una necesidad humana en principio, como resultado del proceso natural vida.
Otro elemento valioso en el presente análisis, responde a la constante humana de adaptación a su medio ambiente, lo que significa que el establecimiento prioritario de la continuidad de la especie, asegura la transacción de la curva de aprendizaje adquirida en el desarrollo del ejercicio de enseñanza formal e informal.

Por tanto, el comportamiento del emprendedor responde a una interacción directa con su entorno, y al constante esfuerzo por mantener la supervivencia de la especie humana, que le permite guiar la orientación cognoscitiva y generación de ideas a la satisfacción de necesidades básicas que evolucionan junto con el individuo, dando generando la creación de empresas e iniciativas productivas de todo tipo, siendo esto parte del proceso natural de la vida del hombre en toda su historia.

Podemos agregar que el ser humano desarrolla una actitud proteccionista o belicista natural condicionado por el ambiente donde habita, todo generado por el celo al querer salvaguardar su espacio y las cosas que siente que le pertenecen por convivir en él. El cuidado de su territorio lo convierte en un ser conflictivo al tratar de proteger su espacio, pues, no está dispuesto a compartir lo que le pertenece con otros seres a los cuales no conoce y teme por 
la pérdida de lo que es suyo, la carrera armamentista de las naciones es uno de los negocios más lucrativos a nivel global para protegerse de los animales salvajes y por supuesto de los individuos al interior de su especie que deseen usurparle su poder o pertenencias; son una resultante ideado para conservar su vida y proteger a los suyos o su territorio con todo lo que ello significa. El hombre en su condición natural de emprendedor, ha llevado esa cualidad a un contexto proteccionista, lo que condiciona su comportamiento a la defensiva de proyectos e ideas, pues no está dispuesto a compartir lo que le pertenece con individuos que son ajenos a la iniciativa, salvo que esos individuos puedan cooperar para hacer del emprendimiento una realidad.
Podemos entonces concluir que el enfoque etológico brinda un acercamiento del comportamiento del ser en el aprovechamiento de los recursos disponibles en su entorno para explotarlos de la mejor manera, otra cosa ha sido que en materia de la evolución, dichas casuísticas se hayan convertido al final en modelos o procesos de negocio de normal desarrollo, con base en la generación de ideas que buscaban incrementar su bienestar, satisfacción de necesidades y aumento de riqueza como un proceso normal del desarrollo.

Acontinuación iniciaremos las aproximaciones al tema desde el enfoque psicológica del emprendedor examinando las características internas y sus competencias.

\section{El enfoque Psicológico ${ }^{4}$}

C. - -

Jontinuando con la revisión literaria desde diferentes visiones sobre el perfil del emprendedor, se encuentra el enfoque psicológico, en donde se concibe que el hombre por naturaleza es un ser comparativo y creador, es parte inherente de su Psique ${ }^{5}$ y siempre ha sido una constante para él preguntarse por qué algunas personas poseen o logran más éxito que otras.

Joseph Schumpeter(1883-1950), pionero en los conceptos de Emprenderismo determina dentro de su modelo teórico que "la verdadera función de un emprendedor es la de tomar iniciativas,

\footnotetext{
${ }^{4}$ Ciencia que estudia los procesos mentales en personas y animales. Real Academia Española, Diccionario de la Lengua Española, Vigésima Segunda Edición. 2001

5 Proveniente del griego y definida como el "alma" era la esencia o fuerza vital de un individuo. Sin embargo para la Psicología actual son los procesos conscientes o inconscientes de la mente humana. (Diccionario terminológico de las ciencias médicas, Salvat Editores. 3ra Edición. 1950)
} 
de crear", lo que brinda al individuo el aprovechamiento de oportunidades del entorno, ello sin que las ideas necesariamente sean producidas por él (Liouville, 2002).

En ese orden de ideasse puede afirmar que el emprendedor es un transformador que percibe gran diversidad de oportunidades en su entorno ${ }^{6}$. Es más, si se analiza lo afirmado por Schumpeter, se encuentra con que el emprendimiento va más relacionado con la acción que transforma, que con el mero ejercicio de generación de ideas. Si lo anterior lo determinamos como la esencia psicológica del emprendedor, nos encontramos que se estaría limitando a competencias relacionadas solamente con la creatividad y la capacidad de análisis, pero existen diferentes autores que determinan otros elementos que a continuación analizaremos.

Dentro de las diversas escuelas de pensamiento del emprendimiento, se han tomado como base algunas de ellas en el proceso de estudio, entre las que se encuentra la Escuela Comportamental y la Escuela Psicológica, representadas por Gartnety Carland (1988) respectivamente. La primera, define al emprendedor por el "conjunto de actividades que pone en marcha para crear una organización", y considera la creación de una empresa como un suceso de contexto basado en el resultado de numerosos fracasos o éxitos, de perseverancia o de informaciones percibidas como importantes ${ }^{7}$.

Por otro lado, Carlant que pertenece a la Escuela Psicológica plantea que "el emprendedor es un individuo con necesidades de cumplimiento, dependencia, gusto por el riesgo y sentimiento por controlar su destino", lo cual es una visión más cognitiva y más personalista basada en las representaciones o pensamientos que el individuo hace de sus comportamientos.

En la misma línea se encuentra a Shaver y Scott (1991), quienes definen al emprendedor como "una persona con un cierto número de atributos psicológicos descritos tanto por la personalidad como por los procesos cognitivos activados por las circunstancias."8 Adicionalmente complementan su concepto afirmando que "es una persona cuya cabeza o mente integra todas las posibilidades, es alguien que cree que la innovación es posible, y tiene la motivación para persistir hasta que el trabajo se haga"(Miner, J. ,1997).

\footnotetext{
6 Emprenderismo, acción gubernamental y academia. Revisión de la literatura. Rodríguez Carlos, Jiménez Manuel. Revista Innovar 15(26) pg. 73 - 89. Universidad Nacional de Colombia. 2005.

Emprenderismo, acción gubernamental y academia. Revisión de la literatura. Rodríguez Carlos, Jiménez Manuel. Revista Innovar 15(26) pg. 73 - 89. Universidad Nacional de Colombia. 2005.
} 
Algunas de las características psicológicas que la literatura propone como básicas para emprender (Sheinberg y Mac Millan,1988; Birley, Westhead, 1994; Westhead y Wright, 1997) son necesidad de logro, necesidad de Independencia y la motivación económica. Lo anterior brinda una preconcepción muy básica acerca del Emprendedor, limitándolo a sólo tres campos, sin embargo otros investigadores han intentado definir un perfil psicológico del emprendedor a fin de diferenciarlos de las otras personas, a continuación se presentan algunas características que son comunes y que han sido revisadas por los diferentes autores.

Perfil Psicológico de los Emprendedores (Barbara V., et.al,2007)

\begin{tabular}{|c|c|}
\hline Caracteristica & Autor año \\
\hline Deseo de independencia & $\begin{array}{l}\text { Anna, chandler, jansen y Mero (200); barredo y } \\
\text { Llorens(1993); Collis, moore y Unwala (1988); Duchéneaut } \\
\text { y Orham (1998); Feesen y Dugan (1989); García y } \\
\text { Wandoseeel(2004); Genesca y venecia(1984); Jenssen } \\
\text { y Kolvered (1992); Koh (1996);Martínez, Sánchez y } \\
\text { Urbina (1998); Rusque (2002); Sheinberg y Mac Millan } \\
\text { (1988);Woo, Cooper y Dunkelberg(1988). }\end{array}$ \\
\hline Gusto por riesgo & $\begin{array}{l}\text { De Pablo y Bueno (2004); Díez de Castro et al (1995); } \\
\text { Douglas y Shepherd (1997); Dubini (1988);Smith, backerd } \\
\text { y Miner (1987). }\end{array}$ \\
\hline Elevada necesidad de logro & $\begin{array}{l}\text { Anna, Chandler, Jansen y mero (2000); Barredo y Llorens } \\
\text { (1993); Box, White y Barr (1993); Collins, Moore y Unwala } \\
\text { (1964); De Pablo y Bueno (2004); Díez de Castro et al } \\
\text { (1995); Dubini (1988); Jenssen y Kolvered(1992); Jonson } \\
\text { (1990);Koh (1990);Koh(1996); McClelland (1968); Rusque } \\
\text { (2002);Woo, cooper y Dunkelberg(1988). }\end{array}$ \\
\hline Alta necesidad de competencia & $\begin{array}{l}\text { Box, White y Barr (1993); Davidsson (1988); De Pablo } \\
\text { y Bueno (2004); Duchéneaut y Orham (1998); Feesen y } \\
\text { Dugan81989) }\end{array}$ \\
\hline Preferencia por la innovación & $\begin{array}{l}\text { Anna, Chandler, Jansen y Mero (2000); De Pablo y } \\
\text { Bueno (2004); Dubini (1988); Garcia y Wandoseel (2004); } \\
\text { Jenssen y Kolvered (1992); Smith, Backerd y Miner (1987) }\end{array}$ \\
\hline
\end{tabular}

Revisando la bibliografía sobre el tema, se puede encontrar que las variables que más se repiten por los diferentes teóricos alrededor del mundo del emprendimiento es la necesidad del logro y el deseo de independencia, las cuales están muy asociadas a las características culturales de un pueblo, a lo que se puede decir que dicha variable debe estar presente en el pensamiento colectivo de una comunidad para que se dé. 
Al analizar las características psicológicas nos podemos encontrar con unos emprendedores con características muy diferentes a los perfiles habituales. (Bhide A., 1979), de la Universidad de Columbia, lo expresa como: "Aquellos que tienen éxito pueden ser gregarios o taciturnos, analíticos o intuitivos, buenos o pésimos para los detalles, adversos al riesgo o sedientos de emociones personales, que delegan, o maníacos del control, pilares de la comunidad o individuos marginales". ( Prats, M,2008).

Se han revisado diferentes escuelas y autores que nos han proporcionado visiones de cómo debería ser un emprendedor, sin embargo, podríamos definir otros perfiles con base en el análisis de competencias ${ }^{8}$, las cuales nos generan preguntas acerca de si se pueden aprender estas competencias, 0 si son solamente innatas. Lo anterior sería similar a preguntarnos si el emprendedor nace o se hace.

El profesor Pablo Cardona ${ }^{9}$ del IESE Business School de la Universidad de Navarra propone un modelo (Cardona P., 2005) ver figura 1 (Prats M.,2008) de competencias que se considera un resumen de las principales competencias que poseen los emprendedores. La ventaja de este modelo es que involucra de manera holística los aspectos del emprendedor y no únicamente características de tipo individual sino también las de entorno.

Figura 1. Las competencias del emprendedor ${ }^{8}$

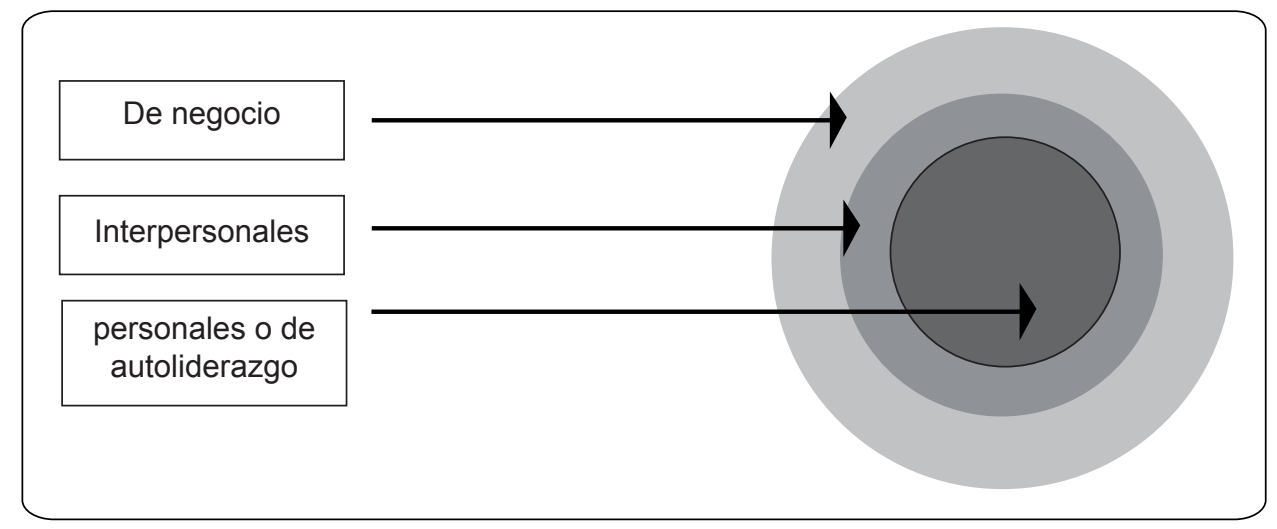

\footnotetext{
${ }^{8}$ Competencias: Conjuntos de conocimientos, habilidades, disposiciones y conductas que posee una persona y que le permiten la realización exitosa de una actividad". Rodríguez y Feliú (1996).

${ }^{9}$ Cardona, Pablo: Professor in the Department of Managing People in Organizations. IESE. Universidad de Navarra. Ph D in Management. U. of California, Los Angeles (UCLA). 1997, Master in Business Administration. (IESE -Universidad de Navarra). 1990. Bachelor's degree in Physical Science. Universidad de Barcelona. 1987.
} 
Se inicia enunciando las competencias de tipo personal que el profesor Cardona incluye en su modelo, estas son: creatividad, determinación, integridad, tenacidad, equilibrio emocional y autocritica. De las anteriores competencias existen dos en la que los diferentes autores siempre han estado de acuerdo: la creatividad y la determinación, características que no solo son reconocidas por los emprendedores sino también por las personas que están a su alrededor y que diríamos que son parte de los elementos por las que las personas se involucran en la idea o negocio que el emprendedor promueve.

Las competencias Interpersonales serian entonces: comunicación, implicación (conocida como carisma), delegación y respeto. De estas competencias consideramos que todas son claves para lograr el desarrollo de ideas y el compromiso delas personas, sinembargo profundicemos en las competencias de Implicación y delegación. La primera es también conocida como carisma el cual implica involucrar a un grupo no importa que el proyecto sea individual. Tener carisma implica que las personas perciban confianza y que sus ideas sean motivadoras para que las personas se puedan comprometer con dicho emprendedor. La delegación implica que cada uno de los participantes tiene la información y los recursos adecuados para tomar decisiones. Es importante aclarar que el emprendedor entrega pero no se exime de la responsabilidad del proyecto.

Las últimas competencias del modelo son las de negocio que comprenden, La Gestión de Recursos, La Visión de Negocios, el Networking y la Negociación. El término mas nuevo sería el de "Networking" que según el autor se refiere a desarrollar y mantener redes de contactos y relaciones claves con relación a la empresa y la industria. Otros autores han evaluado este concepto como "Capital Social" o Redes de Contactos (Hoang y Antoncic 2003; Osgen y Baron 2007; Shane y Cable 2002). En el mundo de hoy con la velocidad de los sistemas y comunicaciones consideramos de vital importancia esta habilidad para lograr identificar las oportunidades y compartir experiencias que disminuyan el sentimiento de incertidumbre ante proyectos nuevos a desarrollar.

Igualmente el termino de visión de negocios es comparable a los términos de alerta empresarial = "Alertness" (kirzner, 1973) o al de conciencia emprendedora = "Entrepreneurial Awareness" (Ray y Cardozo, 1996) los cuales podríamos definir como habilidades del emprendedor para percibir y reconocer oportunidades en el entorno, especialmente identificando las necesidades no satisfechas mediante 
la combinación de recursos novedosos (Alertness, 1973).

Es posible que algunos de los lectores prefieran definir a los emprendedores basados solamente en algunas competencias de cada uno de los pilares del modelo del profesor Cardona, consideramos que ello es válido, debido a que las condiciones culturales y la esencia de cada idea de negocio permiten solamente identificar algunas de ellas, sin embargo el modelo es bastante integral y sirve como guía para entender las características y habilidades que posee un emprendedor.

Continuando con el tema de competencias se considera que una de las principales competencias que un emprendedor debe poseer es la creatividad, tal comolo plantea el profesor Amar Bhidé ${ }^{10}$, que la considera como el "hacer cosas nuevas o hacerlas diferentes creando valor", (Prats, M. y
Agulles, R. ,2008) dicha competencia es generalmente pensada como algo innato que solo lo poseen algunas personas que son consideras por los demás como creativas. Las investigaciones modernas han determinado que "todos los humanos con capacidades normales son capaces de producir por lo menos actividades o trabajos creativos de manera moderada en algún campo, donde muchas veces el entorno social puede influir en el nivel y la frecuencia de este comportamiento creativo. La creatividad es entonces la producción de ideas novedosas y útiles en cualquier área. (Amabile, T., 1996).

Cuáles entonces serían los componentes que conforman la creatividad, la profesora Teresa M. Amabile ${ }^{11}$ (1996) de la Universidad de Harvard (1983a, 1983b, 1988a, 1988b) ha creado un modelo que se considera pertinente presentar:

Figura 2. Modelo de 3 componentes de la creatividad ${ }^{12}$

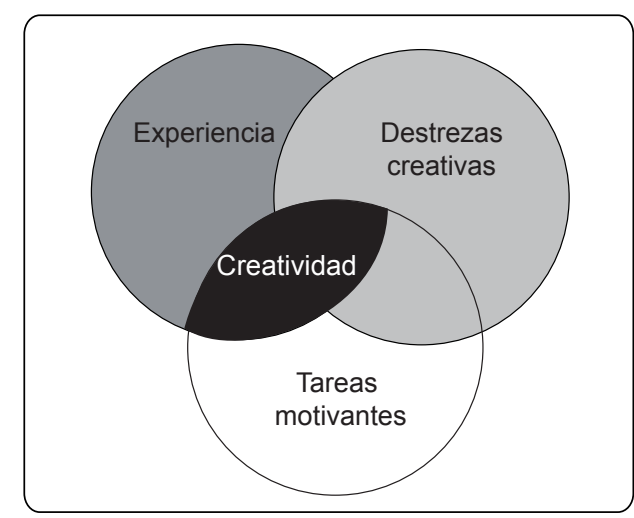

${ }^{10}$ Bhide, Amar. How Entrepreneurs Craft Stategies that Work. Harvard Business Review. Pag 150-161 (1994).

11 Amabile Teresa: Professor of Business Administration in the Entrepreneurial Management Unit at Harvard Business School and Director of Research. Ph.D. in psychology from Stanford University (1977)

12 Amabile, Teresa M. Creativity and innovation in organizations. Harvard Business School. 9-396-239. January 5, 1996. 
Expliquemos entonces los componentes del modelo:

Dxperticia: es el pilar de todo trabajo creativo. Este componente incluye memoria de los hechos conocidos, competencia técnica y talentos especiales del objeto a trabajar.

> Destrezas creativas o pensamientos creativos: incluye un estilo de pensamientos favorables para tomar nuevas perspectivas o problemas en aplicación de técnicas o esquemas para la exploración de nuevos caminos de pensamiento con un estilo de trabajo que conduzca a la persistencia y actividades enérgicas hacia un trabajo.

D Tareas motivantes intrínsecas: $\mathrm{Si}$ las dos anteriores componentes se refieren a lo que un individuo es capaz de hacer en función de lo que domina, el componente de tareas motivacionales determina lo que la persona actualmente haría. La motivación puede tomar dos formas; motivaciones intrínsecas (internas) que son manejadas por profundos intereses personales y las cuales están involucradas en el trabajo, la curiosidad, la diversión o las sensaciones personales de cambio. La otra forma son las motivaciones extrínsecas (externas) que son manejadas por el deseo de alcanzar una meta que se aparte de su propio trabajo, como el logro de una recompensa prometida o ganar un concurso.

La profesora Amabile (1988) también presenta una interesante visión de cómo se integran los componentes del modelo individual de creatividad dentro de un entorno de trabajo organizacional (Ver figura 3). La teoría nos describe como la influencia del entorno organizacional de trabajo en la creatividad de individuos o equipos y a su vez la influencia de individuo y equipos creativos en el global de una organización genera altos estándares de innovación.

De acuerdo con las investigaciones recientes, los emprendedores exitosos en comparación con los administradores, tienden a ser más conscientes, más abiertos a nuevas experiencias, menos neuróticos y más simpáticos o agradables. (Zhao, H \& Seibert, S. 2006) Además de ser más persistentes, trabajadores y organizados, así como de tener un mayor anhelo por el éxito, y un alto grado de responsabilidad. (Collins, C, Hanges, P., \& Locke, E. (2004).

Otras características psicológicas que se han identificado en los últimos 20 años son el alto grado de optimismo, la habilidad social y el uso de atajos cognitivos. Adicional se ha identificado que también muestran menos aversión al riesgo en 
algunos escenarios de incertidumbre donde perciben mayores posibilidades de ganar. Esto no quiere decir que les agrade el riesgo sino que poseen una mejor capacidad para medirlo.

Las características psicológicas del emprendedor permiten inferir que no existe un perfil único con relación a los emprendedores, las características de tipo cultural, económico y personal genera una combinación de factores diferentes que finalmente influyen mas intensamente en algunas personas que en otras para determinar acciones de emprendimiento. Es claro que se debe poseer en mayor medida algunos factores de tipo psicológico al igual que algunas habilidades o competencias para empezar a incursionar en el campo del emprendimiento pero esta regla no es estándar.

Figura 3. Impacto de la organización en el entorno creativo (Amabile.T, 1996)

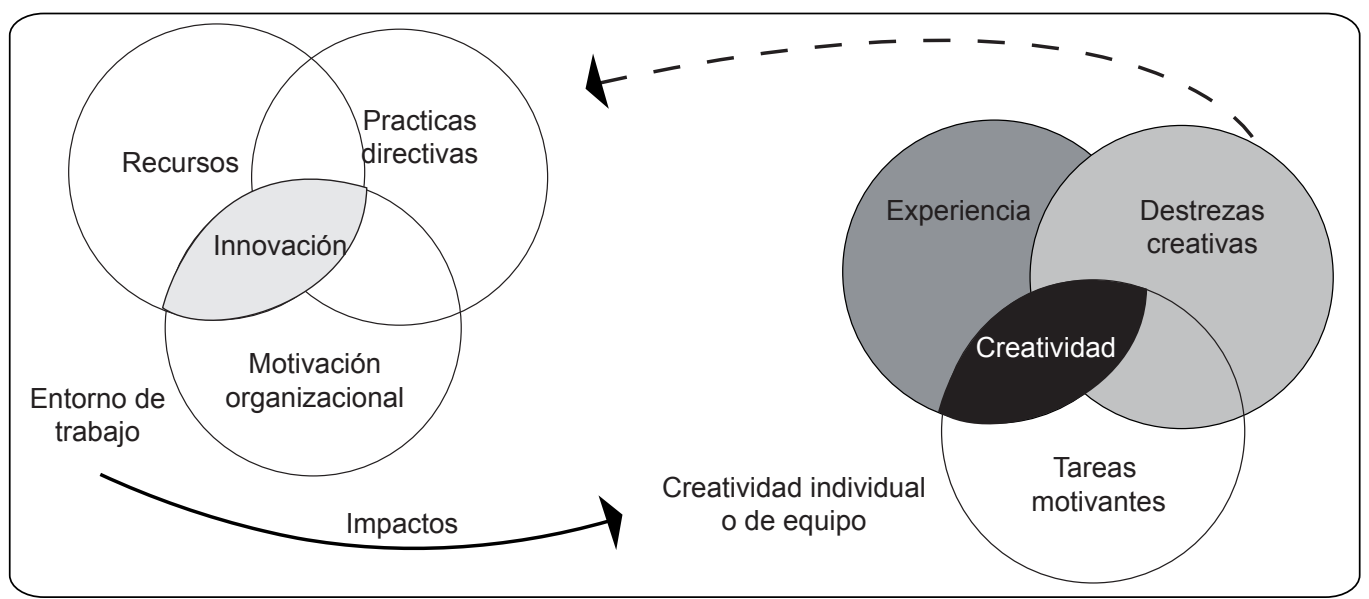




\section{Conclusiones}

No se pretende en una época de cambios socioeconómicos acelerados y en busca de oportunidades de negocios para generar riqueza, poner en entredicho muchas de las más solidas bases del concepto de emprendimiento. Este análisis, resulta de una iniciativa de los autores de desarrollar una idea autónoma de emprendimiento, salida desde las teorías de la etología y la psicología, como tratados del comportamiento del ser humano, como fuente de generación de ideas, y no necesariamente para la creación de empresas.

Se cree, que muchas de las iniciativas de la generación de ideas para el mejoramiento de la economía mundial, no salieron precisamente de la búsqueda de riqueza, si no de los comportamientos innatos de la condición de racionalidad del ser humano, de las necesidades de supervivencia, dependencia y adaptación a su hábitat y sentimiento por defender su propiedad, aunque algunos psicólogos sustentan que las razones provienen más de la satisfacción de necesidades propias del ser: por su individualismo, por la acción en función de un control voluntario, por superación de su dependencia y control por parte del entorno.

A la pregunta sobre si el emprendedor se hace o nace, consideramos que es una mezcla de ambos. El poseer factores internos de personalidad como la motivación el logro, la independencia y creatividad entre otros no son condición única para que una persona desarrolle enfoques emprendedores con su entorno, economía o país. Es precisamente la mezcla de factores culturales, familiares, laborales los que interactúan para potencializar dichas habilidades. Deben existir ambos para que se dé dicho carácter emprendedor. 


\section{Bibliografia}

Perinat, A. (1980): Contribuciones de la etología al estudio del desarrollo Humano y socialización. EL BASILISCO, número 11, noviembre - diciembre.

Diccionario canis.cl. La suma de las pautas de conducta propias de una especie, que le han permitido sobrevivir y llegar hasta nuestros tiempos.

Real Academia Española, Diccionario de la Lengua Española, Vigésima Segunda Edición. 2001.

Diccionario terminológico de las ciencias médicas, Salvat Editores. 3ra . Edición.1950

Rodríguez C. Jiménez M. (2005). Emprendimiento, acción gubernamental y academia. Revisión de la literatura. Revista Innovar 15(26) pg. 73 - 89. Universidad Nacional de Colombia.

Miner, J. (1997). A psychological typology of successful entrepreneurs. Pag. 16. Quorom Books.

Shaver, K. (2004). The cognitive characteristics of the entrepreneur. Pag. 131-137. Sage Publications.

Barba, V. et.al (2007). Effects of entrepreneurial motivation and background on new venture's growth. International Congress "Marketing Trends" Paris, 26th-27th January. Ecole Supérieure de Commerce de parís.

http://www.escpeap.net/conferences/marketing/2007_cp/HTML/pages/strategie. $\mathrm{htm}$

Prats, M. Agulles, R. (2008). Algo más que intuición para los negocios - Competencias que todo emprendedor debería desarrollar. IESE Business School - Universidad de Navarra.DI-765. 
Amabile, T. (1996). Creativity and innovation in organizations. Harvard Business School. 9-396-239.

Zhao, H \& Seibert, S. (2006) The big five personality dimensions and entrepreneurial status. A meta - analytical review. Journal of applied Psychology.

Cardona, P. García P. (2005). Cómo desarrollar las Competencias de Liderazgo. Editorial EUNSA.

Collins, C, Hanges, P., \& Locke, E. (2004). The relationship of achievement motivation to entrepreneurial behavior: A meta - analysis. Human Performance.

Pereira F. (2002). Reflexión sobre algunas características del espíritu emprendedor Colombiano. 